\title{
Rab23 promotes the cisplatin resistance of ovarian cancer via the Shh-Gli-ABCG2 signaling pathway
}

\author{
WENJIE ZHANG ${ }^{1}$, FENG YU ${ }^{2}$, YU WANG $^{3}$, YU ZHANG $^{4}$, LINGXIN MENG $^{1}$ and YUHUA CHI ${ }^{1}$ \\ ${ }^{1}$ Oncology Department, Rizhao People's Hospital, Rizhao, Shandong 276800; ${ }^{2}$ Gynaecology Department, \\ Maternal and Child Health Care and Family Planning Service Center of Rizhao City, Rizhao, Shandong 276826; \\ ${ }^{3}$ Emergency Internal Medicine Department, Central Hospital of Rizhao City; ${ }^{4}$ Blood Drawing Department, \\ Central Blood Station of Rizhao City, Rizhao, Shandong 276800, P.R. China
}

Received March 26, 2017; Accepted December 12, 2017

DOI: $10.3892 / \mathrm{ol} .2018 .7949$

\begin{abstract}
As a novel member of the Rab GTPase family, the role of Rab23 has been reported in multiple types of tumor. However, to the best of our knowledge, the role of Rab23 in ovarian cancer (OC) has not yet been reported. In the present study, immunohistochemistry analysis demonstrated that Rab23 was upregulated in OC tissue; survival analysis indicated that Rab23 expression was associated with a reduced overall survival (OS) rate and disease-free survival (DFS) time. In vitro experiments also demonstrated the increased expression of Rab23 in the OC cells lines, A2780 and SKOV-3, compared with in the normal ovarian cell line, IOSE80. Following the silencing of ABCG2 in SKOV-3 cells, ATP-binding cassette sub-family G member 2 (ABCG2) expression was significantly downregulated both at the RNA and protein levels. The cisplatin (DDP) $\mathrm{IC}_{50}$ declined from $43.09 \pm 7.12 \mu \mathrm{mol} / 1$ in control cells to $26.46 \pm 5.38 \mu \mathrm{mol} / 1$ in SKOV-3 cells with silenced Rab23. In contrast, in A2780 cells overexpressing Rab23 (A2780-Rab23), ABCG2 expression was significantly upregulated and the DDP $\mathrm{IC}_{50}$ increased from $27.42 \pm 6.54 \mu \mathrm{mol} / 1$ in control cells to $45.92 \pm 5.23 \mu \mathrm{mol} / 1$ in A2780-Rab23. Investigation into the potential molecular mechanisms for this revealed that the expression of sonic hedgehog (Shh) and Gli family zinc finger 1 (Gli1) was increased in A2780-Rab23 cells, whereas silencing Rab23 in SKOV-3 cells significantly inhibited the expression of Shh and Gli1. The Gli1 inhibitor GANT-61 significantly abrogated the increased ABCG2 expression in A2780-Rab23 cells. Furthermore, the DDP $\mathrm{IC}_{50}$ in A2780-Rab23 cells decreased significantly following the silencing of ABCG2 expression; the $\mathrm{IC}_{50}$ declined from $51.66 \pm 8.32 \mu \mathrm{mol} / 1$ in $\mathrm{A} 2780-\mathrm{Rab} 23$
\end{abstract}

Correspondence to: Dr Yuhua Chi, Oncology Department, Rizhao People's Hospital, 126 Taian Road, Donggang, Rizhao, Shandong 276800, P.R. China

E-mail: syycyh1234@163.com

Key words: ovarian cancer, Rab23, cisplatin resistance, ATP-binding cassette sub-family G member 2, sonic hedgehog cells to $25.61 \pm 6.17 \mu \mathrm{mol} / 1$ in A2780-Rab23 cells with silenced ABCG2. Collectively, the results indicate that Rab23 promotes the DDP resistance of OC cells via the Shh-Gli1-ABCG2 pathway, providing the proof of principle for the further investigation of drug resistance therapy targeting Rab23.

\section{Introduction}

Ovarian cancer (OC) is the most common type of malignant tumor of the female reproduction system and the leading cause for mortality from gynecological cancer worldwide (1). It is usually characterized by indistinct symptoms and rapid progression, therefore, the majority of patients with OC are diagnosed at an advanced stage and the prognosis is relatively poor (2). In recent years, the overall survival (OS) rate of patients with OC has improved to some extent due to the development and combinations of treatments, including surgery, chemotherapy and molecularly targeted therapy. However, the prognosis for patients with OC remains relatively poor, which is likely attributable to drug resistance $(3,4)$. Therefore, the identification of a novel method for the prevention of OC drug resistance is necessary.

Rab23, a member of the Rab GTPase family, was first isolated from brain tissue in 1994 (5). Its abberant expression has been reported in various different types of tumors; however, its role in tumor progression and its association with prognosis are controversial topics. Jian et al (6) reported that Rab23 could promote squamous cell carcinoma cell migration and invasion via the integrin $\beta 1 / \mathrm{Rac1}$ pathway. Wang et al (7) reported that Rab23 was overexpressed in human astrocytoma and promoted cell migration and invasion through the regulation of Rac1. However, Denning et al (8) demonstrated that Rab23 exhibited low expression and served an inhibitory role in thyroid cancer progression. Furthermore, Liu et al (9) reported that Rab23 expression could inhibit proliferation and promote apoptosis in breast cancer. However, to the best of our knowledge, the expression levels and function of Rab23 in OC has not yet been reported.

In the present study, immunohistochemistry (IHC) analysis demonstrated that Rab23 was highly expressed in OC tissues and positively associated with poor OS and disease-free survival (DFS) time. Cell-level experiments indicated that 
Rab23 was highly expressed in OC cells and could promote the cisplatin (DDP) resistance of OC cells. Investigation into the potential molecular mechanisms behind this effect revealed that the sonic hedgehog (Shh)-Gli family zinc finger 1 (Gli1)-ATP-binding cassette sub-family G member 2 (ABCG2) pathway was potentially responsible for DDP resistance induced by Rab23.

\section{Materials and methods}

Specimens. In total, 74 primary OC specimens were obtained from patients who accepted surgery between January 2008 and December 2010 at Rizhao People's Hospital (Rizhao, China). Following surgery, tissues were immersed in $10 \%$ paraformaldehyde at room temperature for $4 \mathrm{~h}$ and subsequently embedded in paraffin. Patients were aged between 34 and 71 years of age, with a median age of 51 years. Patients who had received chemotherapy, radiotherapy or immunomodulatory therapy prior to surgery were excluded from the study. The present study was approved by the review board and ethics committee of Rizhao People's Hospital.

Immunohistochemistry (IHC). Tissues were cut into 4- $\mu$ m-thick sections and incubated with a rabbit anti-human Rab23 primary antibody (cat. no. ab192420; dilution, 1:300; Abcam, Cambridge, UK) at $4^{\circ} \mathrm{C}$ overnight. Normal rabbit IgG (cat. no. ab172730; dilution, 1:300; Abcam) was used as a negative control. Sections were then incubated with horseradish peroxidase-conjugated goat anti-rabbit IgG (cat. no. KIT-7710; working dilution; Fuzhou Maixin Biotech. Co., Ltd., Fuzhou, China) and stained with 3,3'-diaminobenzidine (1 mg/ml) for $20 \mathrm{sec}$ at room temperature; cell nuclei were stained using $10 \%$ hematoxylin for $14 \mathrm{sec}$ at room temperature. The staining score was calculated by two independent pathologists simultaneously, at x400 magnification in 5 random fields of view. The proportion score represented the percentage of stained tumor cells among total cells in each field of view: $0,<10 \% ; 1,10-25 \%$; $2,26-75 \%$ and $3,>75 \%$. The intensity score represented the overall staining intensity: 0 , none; 1 , weak; 2 , intermediate, and 3, strong. The expression score of Rab23 was calculated as the product of proportion score and intensity score. Scores $\geq 4$ were classified as high expression and $<4$ as low expression.

Cell culture. The IOSE80 human normal ovarian cell line, and OC cell lines A2780 and SKOV-3, were purchased from the American Type Culture Collection (Manassas, VA, USA). All cell lines were cultured in RPMI-1640 medium (Invitrogen; Thermo Fisher Scientific, Inc., Waltham, MA, USA) supplemented with $10 \%$ fetal bovine serum (FBS) (Invitrogen; Thermo Fisher Scientific Inc.). All cells were cultured in $5 \% \mathrm{CO}_{2}$ at $37^{\circ} \mathrm{C}$. GANT61 (cat. no. 500579-04-4, Selleck Chemicals, Houston, TX, USA) is an inhibitor, which can block the hedgehog signaling pathway through inhibiting Gill. This in turn reduces the drug resistance ability of cells. In the present study, GANT61 was used to inhibit Gli1.

Stable transfection. pcDNA3.1/Rab23 or empty plasmids (Shanghai GenePharma Co., Ltd., Shanghai, China) were transfected into A2780 cells to produce A2780-Rab23 cells, and p-GPU6/Rab23-shRNA or empty plasmids (Shanghai
GenePharma Co., Ltd.) were transfected into SKOV-3 cells, using Lipofectamine $2000^{\circledR}$ (Invitrogen; Thermo Fisher Scientific, Inc., Waltham, MA, USA) according to the manufacturer's protocol. Cells were then cultured in $1.0 \mu \mathrm{g} / \mathrm{ml}$ puromycin for 3 weeks and monoclones were selected. Five weeks following transfection, Rab23 expression was detected by RT-PCR and western blotting.

Silencing ABCG2 in OC cells. ABCG2 siRNA (5'-CCAGAA CUAACGCAUUCAAdTdAdAdTGGUCUUGAUUGCGUA AGUU-3'; Shanghai GenePharma Co., Ltd.) was transfected into A2780-Rab23 cells using Lipofectamine ${ }^{\circledR} 2000$ (Invitrogen; Thermo Fisher Scientific, Inc., Waltham, MA, USA), according to the manufacturer's protocol. A negative siRNA was as used as a control. At 48 h, ABCG 2 expression was detected by reverse transcription-quantitative polymerase chain reaction (RT-PCR) and western blotting.

$R T-P C R$. Total RNA was extracted using TRIzol (Life Technologies; Thermo Fisher Scientific, Inc.), according to the manufacturer's protocol. cDNA was synthesized from the total RNA using the PrimeScript RT-PCR kit (Takara Biotechnology Co., Ltd., Dalian, China). The polymerase chain reaction mixture was as follows: $1.2 \mu \mathrm{l} \mathrm{cDNA}, 1.8 \mu \mathrm{l}$ forward primer, $1.4 \mu \mathrm{l}$ reverse primer, $5.6 \mu \mathrm{l}$ double distilled $\mathrm{dH} 2 \mathrm{O}$ was mixed. The thermocycling conditions were as follows: $94^{\circ} \mathrm{C}$ for $5 \mathrm{~min}$, then 30 cycles of $94^{\circ} \mathrm{C}$ for $30 \mathrm{sec}, 54^{\circ} \mathrm{C}$ for $30 \mathrm{sec}, 72^{\circ} \mathrm{C}$ for $30 \mathrm{sec}$ and $72^{\circ} \mathrm{C}$ for $5 \mathrm{~min}$. The products were electrophoretically separated on agarose gel (1.0\%). Bands were analyzed using Labworks software (version 4.0; UVP LLC, Upland, CA, USA). The primer sequences used were as follows: Rab23, forward, 5'-AGGCACTGGCAAAAAGGT TA-3', and reverse, 5'-TAGACCACCTTCAGTGAGGC-3'; ABCG2, forward, 5'-CTGAGATCCTGAGCCTTTGG-3', and reverse, 5'-TGCCCATCACAACATCATCT-3'. GAPDH was used as an internal control and the primer sequence was as follows: GAPDH forward, 5'-AGGTCGGTGTGAACGGAT TTG-3' and reverse, 5'-GGGGTCGTTGATGGCAACA-3'.

Western blot analysis. Total protein concentration was measured using the BCA kit (Pierce; Thermo Fisher Scientific, Inc.) according to the manufacturer's protocol. Radioimmunoprecipitation assay buffer (P0013B; Beyotime Institute of Biotechnology, Haimen, China) containing $1 \%$ protease inhibitor was used for protein extraction. In total, $250 \mu \mathrm{g}$ protein was loaded and separated using SDS-PAGE (10\% gel) and transferred onto nitrocellulose membranes. Blocking was performed using TBS with Tween 20 containing $5 \%$ non-fat dried milk for $1 \mathrm{~h}$ at room temperature. The membranes were incubated with the primary antibodies (Table I) at $4^{\circ} \mathrm{C}$ overnight. The membranes were then incubated with peroxidase-conjugated goat anti-rabbit-IgG (dilution 1:4,000; Abcam, Cambridge, UK) at room temperature for $1 \mathrm{~h}$. Immunoreactive signals were detected using enhanced chemiluminescence reagent (Pierce; Thermo Fisher Scientific, Inc.). The bands were analyzed using Image-Pro (v5.1; Media Cybernetics, Inc., Rockville, MD, USA).

Drug resistance assay. Cells were plated into $96-$ well plates in triplicate at $8 \times 10^{3}$ cells per well. After $24 \mathrm{~h}$, cells were cultured 
Table I. Primary antibodies used in western blotting.

\begin{tabular}{lllc} 
Gene & \multicolumn{1}{c}{ Company } & $\begin{array}{c}\text { Catalogue } \\
\text { number }\end{array}$ & Dilution \\
\hline Rab23 & Abcam, Cambridge, UK & ab192420 & $1: 300$ \\
ATP-binding cassette sub-family G & Abcam, Cambridge, UK & ab24115 & $1: 400$ \\
member 2 & Cell Signaling Technology, Danvers, MA, USA & 2207 & $1: 400$ \\
Sonic hedgehog & Cell Signaling Technology, Danvers, MA, USA & 3538 & $1: 300$ \\
Gli family zinc finger 1 & Abcam, Cambridge, UK & ab9485 & $1: 500$ \\
GAPDH & & &
\end{tabular}

A

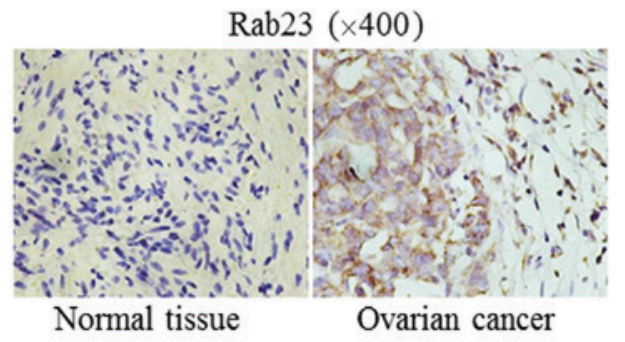

B

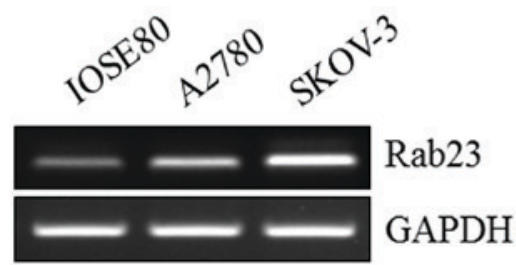

C

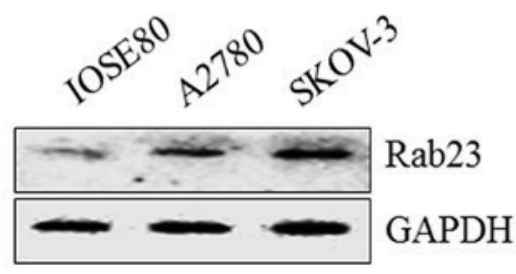

D

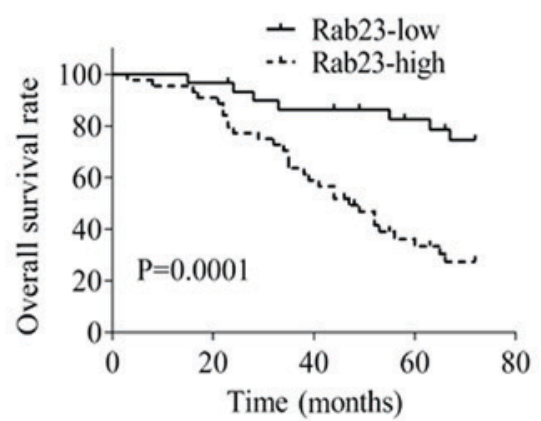

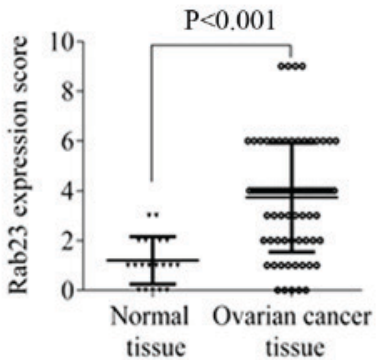
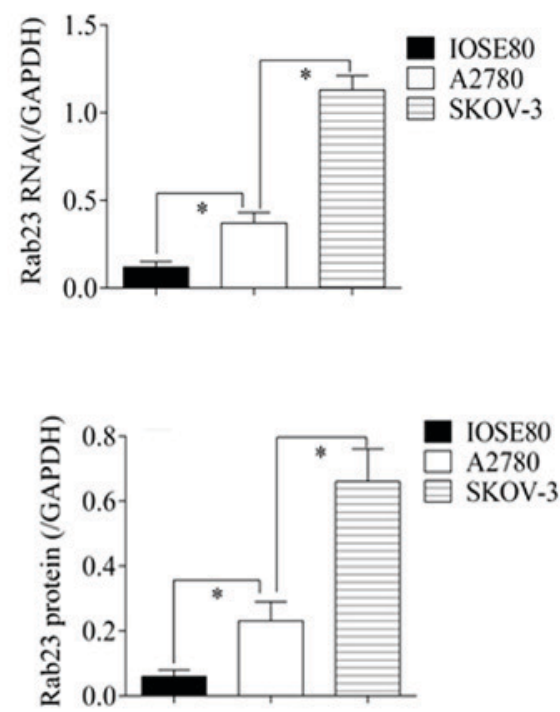

E

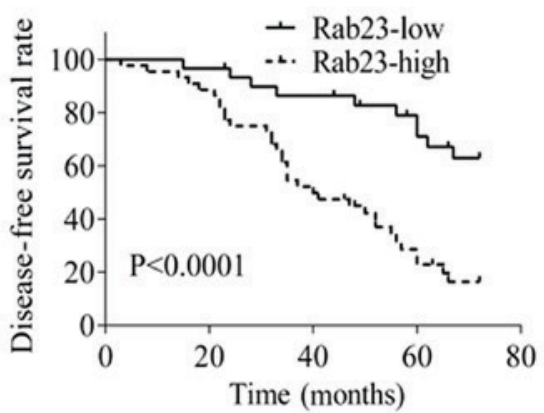

Figure 1. Rab23 is upregulated in OC and negatively associated with overall survival and disease-free survival time. (A) Representative images of the immunohistochemical staining of Rab23 in normal ovarian and OC tissue. Rab23 expression in the normal ovarian cell line, IOSE80, and OC cell lines, A2780 and SKOV-3, at the (B) mRNA and (C) protein levels. (D) Overall and (E) disease free survival analysis of patients with OC, stratified by Rab23 score. ${ }^{*}<0.05$. $\mathrm{OC}$, ovarian cancer. 

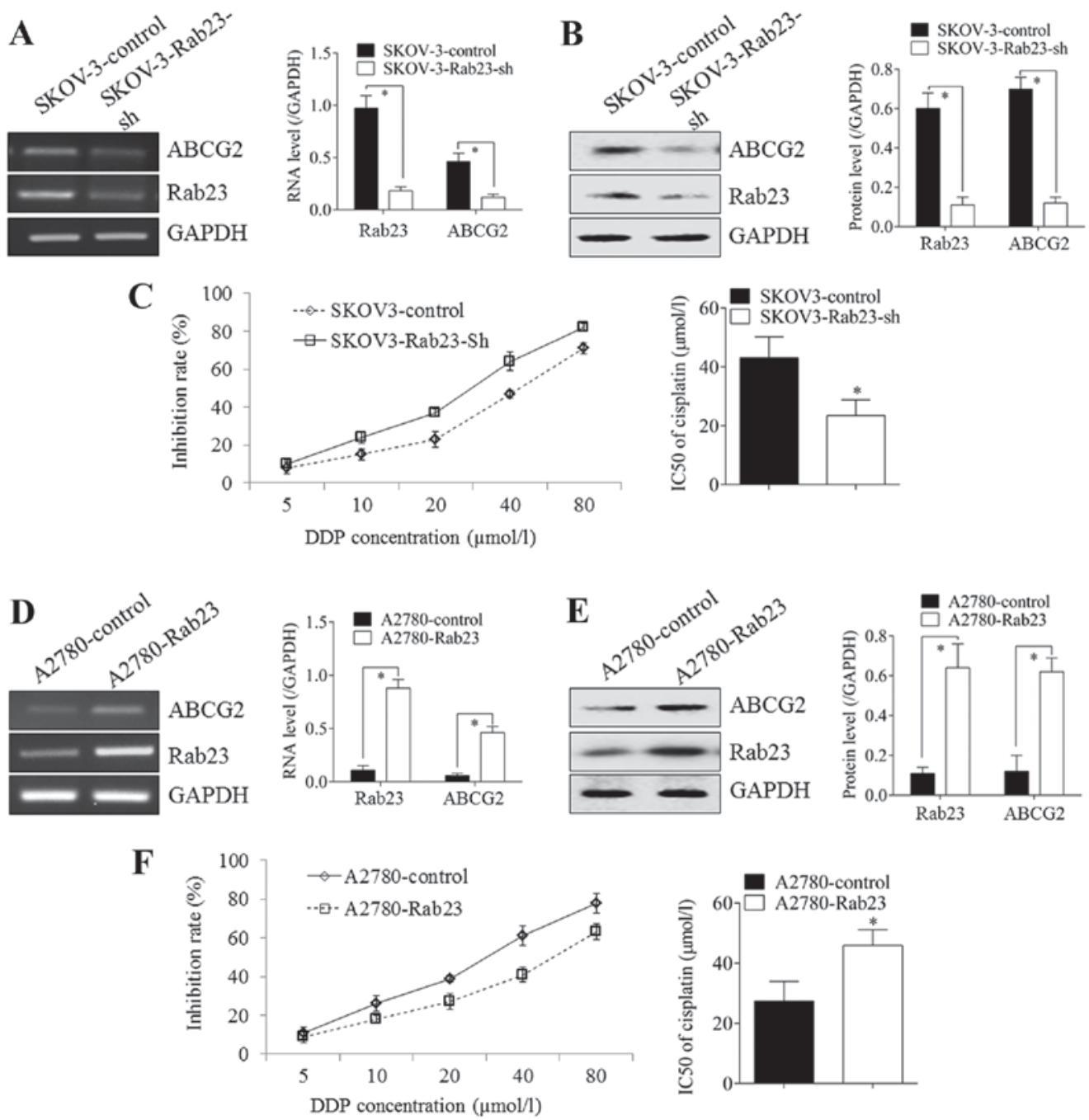

Figure 2. Rab23 expression promotes the expression of ABCG2 and DDP resistance. Rab23 knockdown in SKOV-3 cells resulted in the downregulation of ABCG2 expression at the (A) mRNA and (B) protein levels. (C) Rab23 knockdown caused the growth inhibition rate of cells treated with DDP to increase significantly, with a reduced $\mathrm{IC}_{50}$. (D) Rab23 overexpression in $\mathrm{A} 2780$ cells resulted in the upregulation of $\mathrm{ABCG} 2$ expression at the (D) mRNA and (E) protein levels. (F) Rab23-overexpressing A2780 cells demonstrated a significantly decreased growth inhibition rate when treated with DDP, resulting in a decreased DDP IC 50 value. "P<0.05. ABCG2, ATP-binding cassette sub-family G member 2; DDP, cisplatin.

in medium containing different concentrations of $\operatorname{DDP}(5,10$, 20, 40 and $80 \mu \mathrm{mol} / \mathrm{l})$ for $48 \mathrm{~h}$. Cells were then incubated with MTT for $4 \mathrm{~h}$, then formazan was dissolved with dimethyl sulfoxide. Viability was analyzed by the absorbance at $490 \mathrm{~nm}$. The experiment was repeated at least 3 times. The $\mathrm{IC}_{50}$ was then calculated from the survival curves constructed.

Statistical analysis. All data are expressed as the mean \pm standard deviation. SPSS 11.0 software (SPSS, Inc., Chicago, IL, USA) was used for all statistical analysis. Survival curves were generated by Kaplan-Meier analysis and were compared with the log-rank test. Differences between groups were analyzed using one-way analysis of variance followed by Dunnett's post-hoc test. $\mathrm{IC}_{50}$ was calculated using regression analysis. $\mathrm{P}<0.05$ was considered to indicate a statistically significant difference.

\section{Results}

Rab23 is relatively highly expressed in OC and associated with a reduced $O S$ and DFS rate. Rab23 expression was detected using the IHC staining of 74 OC tissues and 20 normal ovarian control tissues. As demonstrated in Fig. 1A, Rab23 expression in OC tissue was significantly increased compared with normal ovarian tissue $(\mathrm{P}<0.001)$. Cell line experiments also demonstrated that Rab23 expression was higher in the OC cell lines, A2780 and SKOV-3, than in the normal ovarian cell line, IOSE80, at the mRNA (Fig. 1B) and protein (Fig. 1C) levels $(\mathrm{P}<0.05)$.

The 74 OC tissues were divided into low $(n=30)$ and high $(n=44)$ Rab23 expression groups. In the low Rab23 expression group, 7 mortalities occurred during the follow-up period, an OS rate of $76.67 \%$; in the high Rab23 expression group, 30 mortalities occurred, an OS rate of $31.82 \%$. In addition, 10 patients exhibited disease progression in the low Rab23 expression group, a DFS rate of 66.67\%; in the high Rab23 expression group, 34 patients exhibited disease progression, a DFS rate of $22.73 \%$. Using Kaplan-Meier survival curves and the log-rank test, it was determined that the differences in the OS $(\mathrm{P}=0.0001$; Fig. 1D) and DFS rates $(\mathrm{P}<0.0001$; Fig. 1E) were significant. 
A

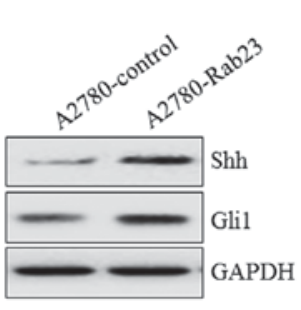

B

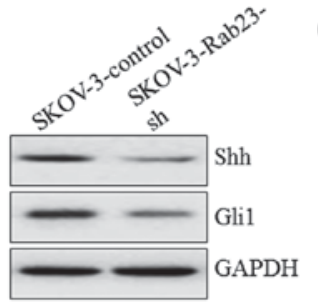

C

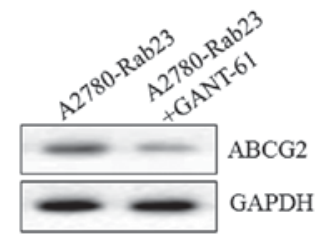

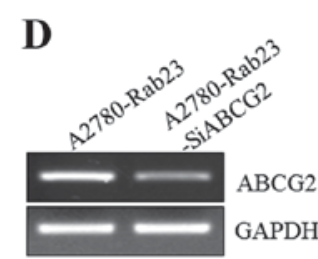
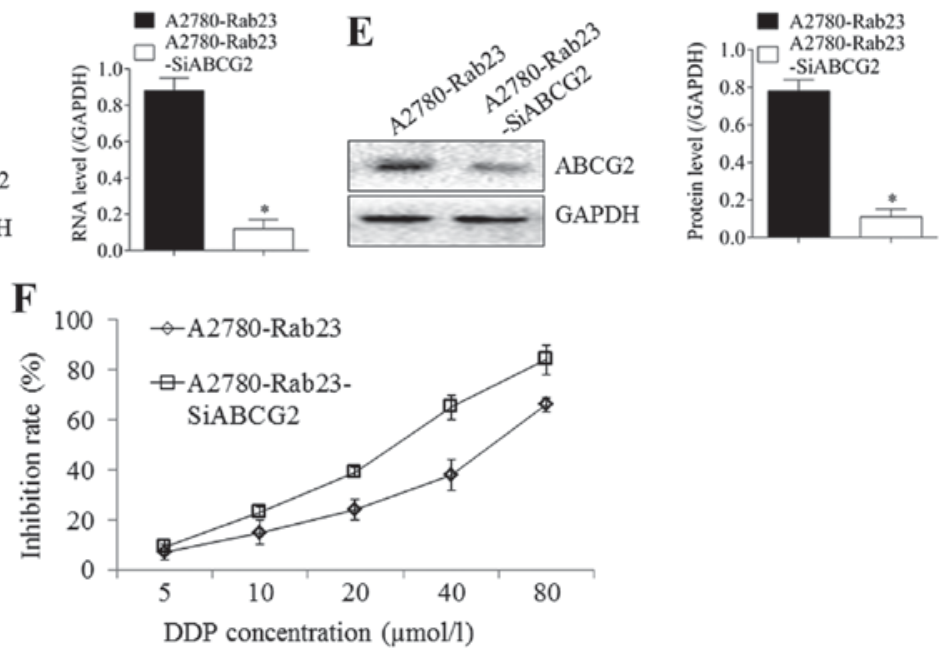

Figure 3. Shh-Gli1-ABCG2 pathway affects the DDP resistance induced by Rab23. (A) Rab23 overexpression in A2780 cells was associated with an increase in Shh and Gli1 protein expression. (B) Silencing Rab23 in SKOV-3 cells caused Shh and Gli1 protein expression to decline. (C) Addition of the Gli1 inhibitor, GANT-61, induced a reduction in ABCG2 expression in A2780-Rab23 cells. The successful knockdown of ABCG2 in A2780-Rab23 cells was demonstrated at the (D) mRNA and (E) protein levels. (F) There was an increase in the growth inhibition of A2780-Rab23 cells upon DDP treatment subsequent to ABCG2 silencing, and the $\mathrm{IC}_{50}$ declined. "P<0.05. Shh, sonic hedgehog; Gli1, Gli family zinc finger 1; ABCG2, ATP-binding cassette sub-family G member 2; DDP, cisplatin.

Rab23 promotes $A B C G 2$ expression and DDP resistance. Silencing Rab23 in SKOV-3 cells caused the downregulation of ABCG2 expression at the mRNA (Fig. 2A) and protein (Fig. 2B) levels. The growth inhibition rate of SKOV-3 cells treated with DDP was significantly higher in Rab23-silenced SKOV3 cells compared with control cells; the $\mathrm{IC}_{50}$ decreased from $43.09 \pm 7.12$ to $26.46 \pm 5.38 \mu \mathrm{mol} / 1$ (Fig. 2C). Rab23 overexpression in A2780 cells resulted in the upregulation of ABCG2 expression at the mRNA (Fig. 2D) and protein (Fig. 2E) levels. The growth inhibition rate of A2780 cells treated with DDP was significantly lower in RAb23-overexpressing A2780 cells compared with control cells; the $\mathrm{IC}_{50}$ increased from $27.42 \pm 6.54$ to $45.92 \pm 5.23 \mu \mathrm{mol} / \mathrm{l}($ Fig. 2F; P<0.05).

Shh-Gli1-ABCG2 is associated with the DDP resistance induced by Rab23 expression. Rab23 overexpression in A2780 cells caused the Shh and Gli1 protein expression to increase compared with empty control plasmid-transfected A2780 cells (Fig. 3A). The silencing of Rab23 in SKOV-3 cells resulted in the downregulation of Shh and Glil protein expression compared with empty control plasmid-transfected SKOV-3 cells (Fig. 3B). Furthermore, the addition of the Gli1 inhibitor, GANT-61, to Rab23-overexpressing A2780 (A2780-Rab23) cells resulted in a significant decrease in ABCG2 expression compared with untreated A2780-Rab23 cells (Fig. 3C). Silencing ABCG2 in A2780-Rab23 cells (Fig. 3D and E) significantly reduced the inhibition rate induced by Rab23 overexpression compared with un-transfected A2780-Rab23 cells $(\mathrm{P}<0.05)$; the $\mathrm{IC}_{50}$ declined from $51.66 \pm 8.32$ to $25.61 \pm 6.17 \mu \mathrm{mol} / 1$ (Fig. 3F). These results suggest that the Shh-Gli1-ABCG2 pathway was induced by $\mathrm{Rab} 23$ to promote drug resistance.

\section{Discussion}

As a member of the Ras-related small GTPase family, Rab23 has been studied in various types of tumor, and whether it has a negative or positive role in tumor progression is a controversial issue (6-9). In recent years, tumor-promoting roles for Rab23 have been reported in several types of tumors. In 2015, Cai et al (10) reported that Rab23 activated the invasion and motility of pancreatic duct adenocarcinoma cells. In 2017, Chang et al (11) reported that the downregulation of Rab23 in prostate cancer could inhibit tumor growth in vitro and in vivo. However, the role of Rab23 in ovarian cancer remains unknown. In the present study, the relatively high expression of Rab23 was demonstrated in OC tissue and cell lines. Survival analysis indicated that Rab23 was positively associated with the poor OS and DFS rates of OC patients. This is consistent with the studies by Cai et al (10) on pancreatic duct adenocarcinoma, and by Chang et al (11) on prostate cancer. However, the results are conflicting with studies in thyroid and breast cancer $(8,9)$. This may be associated with the different origins of the tumors.

ABCG2, also known as breast cancer resistance protein, is a member of the $\mathrm{ABC}$ family of efflux proteins and can transport substrates out of cells, resulting in drug resistance $(12,13)$. 
Thus, ABCG2 is considered an important target for drug resistance research, and ABCG2 inhibitors are being assessed in clinical studies. In the present study, the silencing or overexpression of Rab23 induced the down- or upregulation of ABCG2 expression, respectively, at the mRNA and protein levels. DDP is one of the most commonly used chemotherapy drugs in multiple types of tumors, and functions by inducing cancer cell death via the activation of the DNA damage response, leading to cell cycle arrest and the induction of mitochondrial apoptosis $(14,15)$. In OC, DDP is used as a first line chemotherapy drug (16). In the present study, it was demonstrated that the DDP resistance of OC diminished when Rab23 was silenced. Overall, these results indicate that Rab23 functions in the drug resistance mechanism of OC cells.

The Shh signaling pathway is an important regulator of human cell proliferation, differentiation and embryonic development (17). The dysregulated activation of the Shh signaling pathway in cancer cells is associated with tumor occurrence, drug resistance and poor cancer prognosis (18). Gli1 activates the target genes downstream of the Shh pathway $(19,20)$. It has been reported that Rab23 is associated with the Shh signaling pathway, but whether this correlation is positive or negative is still under debate. Chi et al (21) reported that Rab23 could negatively regulate Gli1 in a SUFU-dependent manner. However, Fuller et al (22) reported that Rab23 promotes nodal signaling in vertebrate left-right patterning independent of the Shh signaling pathway. In the present study, it was demonstrated that Rab23 upregulated ABCG2 expression via the Shh-Gli signal pathway in OC cells. The differences in the results regarding the effect of Rab23 on Shh signaling between studies may be attributable to the different types of cell under investigation.

In conclusion, the present study demonstrates that Rab23 may function in the drug resistance of $\mathrm{OC}$, and presents a novel potential molecular mechanism for this effect. The present study provides experimental evidence to support the further investigation of Rab23 as a target to inhibit drug resistance and improve the prognosis of patients with OC.

\section{Competing interests}

The authors declare that they have no competing interests.

\section{References}

1. Huo J, Bian XH, Huang Y, Miao ZC and Song LH: Inhibitory effect and mechanism of metformin on human ovarian cancer cells SKOV-3 and A2780. Eur Rev Med Pharmacol Sci 21: 484-489, 2017.

2. Li R, Dong T, Hu C, Lu J, Dai J and Liu P: Salinomycin repressed the epithelial-mesenchymal transition of epithelial ovarian cancer cells via downregulating Wnt/b-catenin pathway. Onco Targets Ther 10: 1317-1325, 2017.

3. Perroud HA, Scharovsky OG, Rozados VR and Alasino CM: Clinical response in patients with ovarian cancer treated with metronomic chemotherapy. Ecancermedicalscience 11: 723, 2017.
4. Lee HH, Bellat V and Law B: Chemotherapy induces adaptive drug resistance and metastatic potentials via phenotypic CXCR4-expressing cell state transition in ovarian cancer. PLoS One 12: e0171044, 2017.

5. Chen Y, Ng F and Tang BL: Rab23 activities and human cancer-emerging connections and mechanisms. Tumour Biol 37: 12959-12967, 2016.

6. Jian Q, Miao Y, Tang L, Huang M, Yang Y, Ba W, Liu Y, Chi S and $\mathrm{Li} \mathrm{C}$ : Rab23 promotes squamous cell carcinoma cell migration and invasion via integrin b1/Racl pathway. Oncotarget 7: 5342-5352, 2016

7. Wang M, Dong Q and Wang Y: Rab23 is overexpressed in human astrocytoma and promotes cell migration and invasion through regulation of Rac1. Tumour Biol 37: 11049-11055, 2016.

8. Denning KM, Smyth PC, Cahill SF, Finn SP, Conlon E, Li J, Flavin RJ, Aherne ST, Guenther SM, Ferlinz A, et al: A molecular expression signature distinguishing follicular lesions in thyroid carcinoma using preamplification RT-PCR in archival samples. Mod Pathol 20: 1095-1102, 2007.

9. Liu Y, Zeng C, Bao N, Zhao J, Hu Y, Li C and Chi S: Effect of Rab23 on the proliferation and apoptosis in breast cancer. Oncol Rep 34: 1835-1844, 2015.

10. Cai ZZ, Xu LB, Cai JL, Wang JS, Zhou B and Hu H: Inactivation of Rab23 inhibits the invasion and motility of pancreatic duct adenocarcinoma. Genet Mol Res 14: 2707-2715, 2015.

11. Chang J, Xu W, Liu G, Du X and Li X: Downregulation of Rab23 in prostate cancer inhibits tumor growth in vitro and in vivo. Oncol Res 25: 241-248, 2017.

12. Nishihashi K, Kawashima K, Nomura T, Urakami-Takebayashi Y, Miyazaki M, Takano M and Nagai J: Cobalt chloride induces expression and function of breast cancer resistance protein (BCRP/ABCG2) in human renal proximal tubular epithelial cell line HK-2. Biol Pharm Bull 40: 82-87, 2017.

13. Wang J, Yunyun Z, Wang L, Chen X and Zhu Z: ABCG2 confers promotion in gastric cancer through modulating downstream CRKL in vitro combining with biostatistics mining. Oncotarget 8: 5256-5267, 2017.

14. Kuo WY, Hwu L, Wu CY, Lee JS, Chang CW and Liu RS: STAT3/NF-kB-regulated lentiviral TK/GCV suicide gene therapy for cisplatin-resistant triple-negative breast cancer. Theranostics 7: 647-663, 2017.

15. Maeda O, Matsuoka A, Miyahara R, Funasaka K, Hirooka Y, Fukaya M, Nagino M, Kodera Y, Goto H and Ando Y: Modified docetaxel, cisplatin and capecitabine for stage IV gastric cancer in Japanese patients: A feasibility study. World J Gastroenterol 23: 1090-1097, 2017.

16. Xie XQ, Zhao QH, Wang $\mathrm{H}$ and Gu KS: Dysregulation of mRNA profile in cisplatin-resistant gastric cancer cell line SGC7901. World J Gastroenterol 23: 1189-1202, 2017.

17. Du Z, Zhou F, Jia Z, Zheng B, Han S, Cheng J, Zhu G and Huang P: The hedgehog/Gli-1 signaling pathways is involved in the inhibitory effect of resveratrol on human colorectal cancer HCT116 cells. Iran J Basic Med Sci 19: 1171-1176, 2016.

18. Merchant JL and Ding L: Hedgehog signaling links chronic inflammation to gastric cancer precursor lesions. Cell Mol Gastroenterol Hepatol 3: 201-210, 2017.

19. Lee HJ, Wu Q, Li H, Bae GU, Kim AK and Ryu JH: A sesquiterpene lactone from Siegesbeckia glabrescens suppresses Hedgehog/Gli-mediated transcription in pancreatic cancer cells. Oncol Lett 12: 2912-2917, 2016.

20. Khatra H, Kundu J, Khan PP, Duttagupta I, Pattanayak S and Sinha S: Piperazic acid derivatives inhibit Gli1 in Hedgehog signaling pathway. Bioorg Med Chem Lett 26: 4423-4426, 2016.

21. Chi S, Xie G, Liu H,Chen K, Zhang X,LiC and Xie J: Rab23 negatively regulates Gli1 transcriptional factor in a $\mathrm{Su}(\mathrm{Fu})$-dependent manner. Cell Signal 24: 1222-1228, 2012.

22. Fuller K, O'Connell JT, Gordon J, Mauti O and Eggenschwiler J: Rab23 regulates Nodal signaling in vertebrate left-right patterning independently of the Hedgehog pathway. Dev Biol 391: 182-195, 2014. 